\title{
Genetic linkage analysis of DFNB22 in families with autosomal recessive non-syndromic hearing loss in Khuzestan province
}

\author{
Ladan Sadeghian ${ }^{1}$, Mohammad Amin Tabatabaiefar ${ }^{2,3}$, Morteza Hashemzadeh Chaleshtori ${ }^{1 * \mathbb{D}}$ \\ ${ }^{1}$ Cellular and Molecular Research Center, Basic Health Sciences Institute, Shahrekord University of Medical Sciences, \\ Shahrekord, Iran \\ ${ }^{2}$ Department of Genetics and Molecular Biology, School of Medicine, Isfahan University of Medical Sciences, Isfahan, Iran \\ ${ }^{3}$ Pediatric Inherited Diseases Research Center, Research Institute for Primordial Prevention of Non-communicable Disease, \\ Isfahan University of Medical Sciences, Isfahan, Iran
}

*Corresponding Author: Morteza Hashemzadeh Chaleshtori: Cellular and Molecular Research Center, Basic Health Sciences Institute, School of Medicine, Shahrekord University of Medical Sciences, Shahrekord, Iran. Tel: +98 383 3335654; fax: +98 383 3330709; Email: mchalesh@yahoo.com

\begin{abstract}
Background and aims: Hearing loss $(\mathrm{HL})$ is the most common sensorineural disorder affecting 1 in 1000 newborns. Autosomal recessive non-syndromic hearing loss (ARNSHL), which is the most common cause of severe $\mathrm{HL}$, is caused by mutations in more than 80 loci. The OTOA gene located on DFNB22 is a rare cause of the disease and the gene studied less in Iranian ARNSHL families. Hence, limited information is available on the frequency and type of OTOA mutations in different populations. In this study, we investigated the role of DFNB22 locus in ARNSHL patients in Khuzestan province, Iran.

Materials and Methods: In this descriptive-experimental study, 23 large families with pre-lingual ARNSHL from Khuzestan province were enrolled. Mutations in GJB2 were excluded by DNA sequencing followed by linkage analysis. Homozygosity mapping of DFNB22 was conducted using 6 short tandem repeat polymorphic markers via touch-down PCR and polyacrylamide gel electrophoresis. Homozygosityby-descent was identified by calculating two-point and multi-point LOD score and haplotype reconstruction.

Results: Families were negative for GJB2 mutations. Genotyping the STRP markers, haplotype reconstruction, and two-point and multiplepoint LOD scores did not show homozygosity-by-descent in any of the pedigrees.

Conclusion: Our findings suggest that OTOA mutations might not contribute significantly to the molecular pathophysiology of ARNSHL in Khuzestan province. However, extending the sample size can illuminate the role of this gene in Khuzestan province.

Keywords: Genetic linkage analysis; ARNSHL; DFNB22; OTOA.
\end{abstract}

Received: 01 November 2019, Accepted: 28 November 2019, ePublished: 30 October 2019

\section{Introduction}

Hearing loss (HL) is the most common sensorineural disorder with more than 400 million people affected worldwide. According to the epidemiological studies, 1 per 1000 infants suffers from severe to profound HL (1). Communication and language difficulties are the consequences of a delayed diagnosis (2).

HL could occur due to genetic and environmental factors or a combination of both. It is estimated that $50 \%$ of the patients are genetically defective. Non-syndromic HL includes more than $70 \%$ of the cases, while the syndromic form is a rare condition which comprises about $30 \%$ of the patients (3). Autosomal recessive non-syndromic HL (ARNSHL) is the most common form of hereditary HL $(1,4)$. To date, about 90 distinct loci and 60 genes have been identified for ARNSHL as a highly heterogeneous condition (5).
The high rate of consanguinity increases the incidence of recessive genetic disorders. Hence, Iran with an average rate of $38.6 \%$ includes families with rare conditions in comparison with other parts of the world (6). HL accounts for the second most common cause of disability in Iran.

Mutations in the GJB2 and GJB6 genes, located on DFNB1A/B, have been targeted in most studies in Iran. The prevalence of GJB2 mutations varies among different Iranian ethnicities, with the highest frequency of mutations in the northern regions of Iran and the absence of mutations in southern provinces like Sistan and Baluchestan (7). Elucidating the role of other DFNB loci in each of ethnic populations of Iran could give us a better insight for genetic diagnosis of ARNSHL in the future.

Genetic linkage analysis is a powerful method to investigate genetically heterogeneous diseases in large families. The method is based on autozygosity mapping,

(C) 2019 The Author(s); Published by Shahrekord University of Medical Sciences. This is an open-access article distributed under the terms of the Creative Commons Attribution License (http://creativecommons.org/licenses/by/4.0), which permits unrestricted use, distribution, and reproduction in any medium, provided the original work is properly cited. 
which was first proposed by Botstein and Lander (8).

The OTOA gene is located on DFNB22 locus and contains 28 exons and encompasses $82.2 \mathrm{~kb}$ region of the genome. The OTOA gene produces the otoancorin with 1140 amino acids. This protein is mainly expressed on the apical surfaces of the polar epithelial cells located within the inner ear and has no similarity to non-collagen glycoproteins of acellular gels in the inner ear. It appears that this protein is involved in the attachment of acellular gels to the sensory and non-sensory cells of the inner ear (9).

About 17 pathogenic variants have been identified in previous studies throughout the world (10). Hence, limited information is available on the frequency and type of mutations in the $O T O A$ gene in different populations. Here we aimed to investigate the role of DFNB22 in molecular pathology of ARNSHL in the GJB2-negative families.

\section{Materials and Methods}

Subjects and sampling

The present descriptive-experimental study was conducted in Cellular and Molecular Research Center of Shahrekord University of Medical Sciences. The study was conducted on 23 large inbred families with about 120 ARNSHL individuals recruited from Khuzestan province. Clinical investigations including skeletal, eye, kidney, heart, and cutaneous examination were performed. MRI and CT scan were conducted to detect inner ear malformations. Deafness severity was proved by pure-tone audiometry at frequencies ranging from $250 \mathrm{~Hz}$ to $8000 \mathrm{~Hz}$.

\section{Genomic DNA extraction}

After obtaining informed written consent from patients or their parents, $5 \mathrm{~mL}$ of peripheral blood was collected in EDTA-containing tubes. DNA was extracted by the phenol-chloroform method and its quantity and quality were measured via Nanospec cube biophotometer (Nanolytik:, Dusseldorf, Germany) and 1\% agarose gel electrophoresis (11).

\section{The mutation analysis GJB2 gene}

At least, one patient from each family underwent mutation analysis of the GJB2 gene through PCR-sequencing using forward: 5'-CTCCCTGTTCTGTCCTAGC-3' and reverse: 5'-CTCATCCCTCTCATGCTGTC-3' primers. In addition, genetic linkage analysis of DFNB1A/B was performed by short tandem repeat polymorphic (STRP) markers to rule out regulatory and non-coding mutations of the GJB2 gene and mutations of the GJB6 gene.

\section{Genetic linkage analysis of the OTOA gene}

Simulation analysis was performed using FastSLink software version 2.51 to evaluate suitable families for genetic linkage analysis. Six STRP markers were selected via NCBI Genome Browser and UCSC Map Viewer databases. The criteria for selecting markers were the distance from the gene and their polymorphic content. In the case of uninformative markers, other markers close to the gene were replaced. The primers were designed by primer3 version 0.4 .0 (http://bioinfo. ut.ee/primer3-0.4.0/) and primer specificity was checked by NCBI primer blast. Touch-down polymerase chain reaction (PCR) was applied under standard condition to amplify STRP markers. Primer sequences are presented in Table 1.

Each microtube for PCR contained $2.5 \mu \mathrm{L}$ of PCR buffer (10X), $0.5 \mu \mathrm{L}$ of $\mathrm{MgCL}_{2}(50 \mathrm{mM}), 0.5 \mu \mathrm{L}$ of dNTP (10 $\mathrm{mM}), 0.5 \mu \mathrm{L}$ of each primer (10 pM), $1 \mu \mathrm{L}$ of DNA (40-50 ng), and $0.1 \mu \mathrm{L}$ of Taq polymerase, and distilled water was added to reach a final volume of $25 \mu \mathrm{L}$. Standard cycling conditions were performed in a thermocycler (ASTEC PC-818; ASTEC, Fukuka, Japan). The touch-down PCR program was as follows: the initial denaturation at $95^{\circ} \mathrm{C}$ for 3 minutes, 8 cycles of $95^{\circ} \mathrm{C}$ for 30 seconds, $60^{\circ} \mathrm{C}$ for 30 seconds in the first cycle with $1^{\circ} \mathrm{C}$ reduction per cycle, and $72^{\circ} \mathrm{C}$ for 25 seconds, 27 cycles of $95^{\circ} \mathrm{C}$ for 30 seconds,

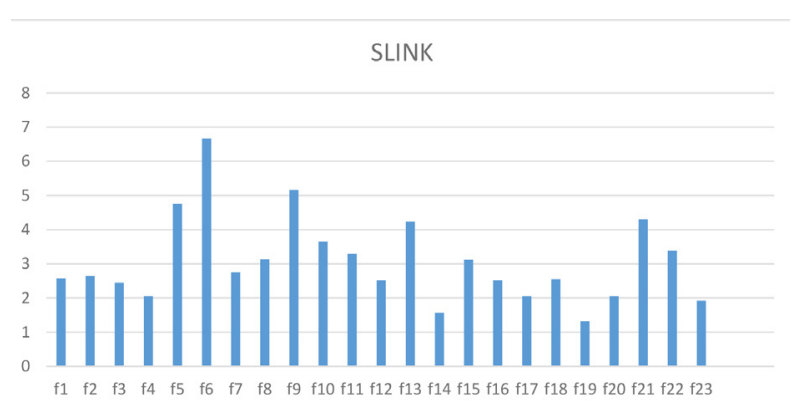

Figure 1. The LOD scores of the families.

Note. The horizontal axis represents the families and the vertical axis represents the SLINK value.

Table 1. Markers used for DFNB22 locus and Their Primers

\begin{tabular}{lcc}
\hline Name & Forward primer & \multicolumn{1}{c}{ Reverse primer } \\
\hline D16S3045 & ATGTAGNACGGCTGAATGTC & GGCCAGATCACATAGTCTCA \\
D16S3046 & CCCAGAATAAACTGCGTG & TTCATGGACCCCCTATTG \\
D16S403 & GTCACCAGATTGCATGTTTT & TATTCATTTGTGTGGGCATG \\
D16S3099 & GAGTAGCATCTCACAACATCTCAC & ACTTTACCCTTAGGGCTCTCC \\
D16S417 & TCCTTCTTCCCATAACAACA & GGATAATGAGTCTCTCTTTCCC \\
D16S3041 & AGTCCCTGCCTTGGTTAGTT & CAGCCTAGGTGACAGAGAAA \\
\hline
\end{tabular}




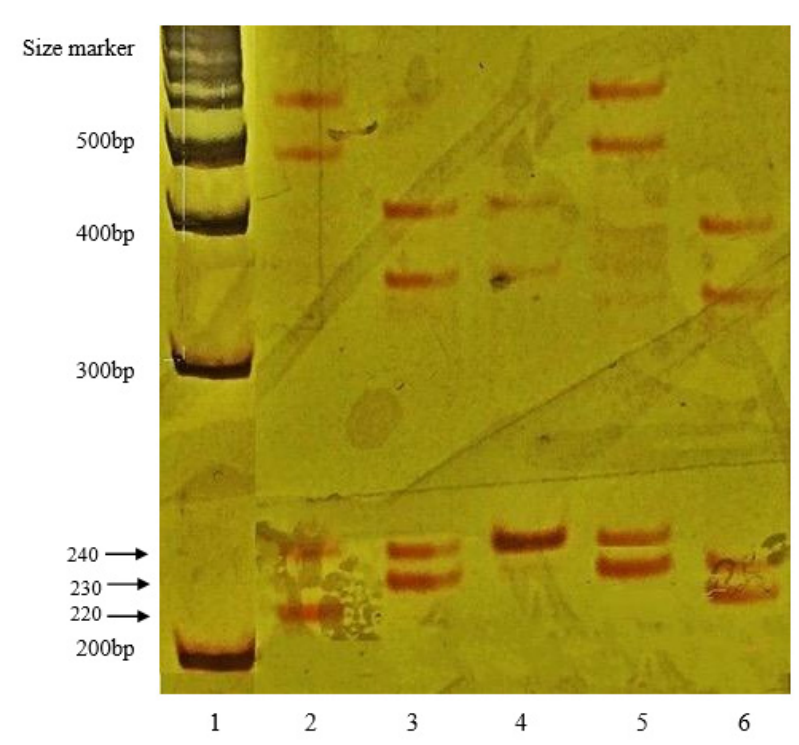

Figure 2. The D16S3045 marker.

Note. Columns represent the genotype of an example family, 1 : ladder $100 \mathrm{bp}$, 2: father, 3: mother, 4, 5: HL offspring, 6: healthy offspring. This marker showed homozygous pattern only in one of the deaf children, although the other was heterozygous.

$53^{\circ} \mathrm{C}$ for 30 seconds, $72^{\circ} \mathrm{C}$ for 25 seconds and one cycle of the final extension at $72^{\circ} \mathrm{C}$ for 10 minutes.

PCR products were resolved on a non-denaturing 8-12\% polyacrylamide gel and stained by silver nitrate. Genotyping was done by visual inspection. Haplotype reconstruction was carried out by HaploPainter version 1.043 (12).

The two-point and multipoint parametric LOD scores were computed respectively by SuperLink version 1.6 and SimWalk version 2.91. The haplotype map was plotted using the HaploPainter software version 1.043 to confirm or reject homozygosity.

\section{Results}

The current study investigated 23 DFNB1A/B-negative families. Pedigree analysis and clinical examination outcomes suggested the presence of ARNSHL. The majority of deaf individuals suffered from severe to profound bilateral sensorineural HL. The values of the LOD scores of the families were estimated to be 1.5-7 (Figure 1).

The mutation analysis of GJB2 and homozygosity mapping of DFNB1A/B revealed no mutation or disease association. STRP genotyping and haplotyping were repeated at least twice for each family. Haplotype analysis and LOD scores did not show homozygosity-by-descent at DFNB22 in any of the families (Figure 2). An example of the plotted haplotype is shown in Figure 3.

\section{Discussion}

HL is a genetically heterogeneous disorder. It is estimated that up to $1 \%$ of human genes are involved in auditory processing (5). However, in most studies, researchers investigated the most prevalent genes and determined their

\section{Family SHO-5}

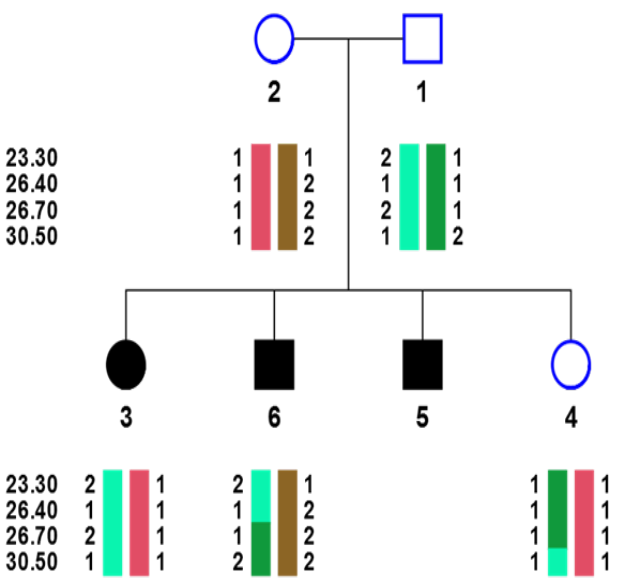

Figure 3. Haplotypes represent lack of genetic linkage in one of the studied families. In this pedigree, in patients with different allelic haplotypes, there is no association between $\mathrm{HL}$ and this locus in this family. The genetic map of the markers is based on Marshfield.

role in the incidence of HL. The GJB2 gene, which has contributed to $16-18 \%$ of ARNSHL in Iran, is known to be the first cause of the disease throughout the world $(13,14)$. Subsequently, SLC26A4 located on DFNB4 (accounting for about 10\%) and TECTA located on DFNB21 (accounting for approximately 4\%) are the most common genes involved in ARNSHL in Iran (12).

A significant percentage of disease-causing genes remain unknown, suggesting the need for further genomic studies to uncover the disease etiology.

In this study, we examined the contribution of DFNB22 in 23 families with at least three deaf members in Khuzestan province in order to complete the studies on ARNSHL. The first study on this locus was conducted in 2002 by Zwaenepoel et al on 200 large Palestinian consanguineous families, which led to identification of DFNB22 in 16p13.1-q11.2 chromosome region carrying the OTOA gene. The IVS12+2T-C mutation in splice site of this gene was identified in one of the families (15).

In another study, Shahin et al in 2010 identified a homozygous $500 \mathrm{~kb}$ deletion that resulted in complete deletion of this gene. In the three Pakistani families, two different heterozygous substitution mutations (G451D, P627S) were identified in this gene. Although no functional studies have been carried out to investigate the effect of these two mutations, it is presumed that both would lead to a disruption of the super-helical structure of the otoancorin (16). Another Pakistani family showed two transient mutations causing disease: c. $1352 \mathrm{G}>\mathrm{A}$ in exon 13 and c.1879C $>\mathrm{T}$ in exon 17 (17).

Investigating 300 families with Chinese, Caucasian, Israeli, and Spanish origins in separate studies showed no linkage to this locus $(18,19)$. 
No mutation in this gene has been reported in Iran until now. In a study on 41 large families with HL, no family showed linkage to this locus (20).

Another study on 67 families from different provinces of Iran reported no linkage to this locus (21). Further, a survey on 144 families from different Iranian populations revealed no mutation in this locus (22).

In the present study, considering the fact that we investigated 23 large families with high consanguinity rates, there was no linkage to this locus, confirming other studies in Iran, all of which relate to the insignificant role of this locus in the incidence of HL. The cause of HL in these patients is likely to be loci involved with a higher incidence. To determine the role of this locus in developing HL, it is necessary to extend studies to other areas and families in order to examine its role more precisely.

\section{Conclusion}

According to the results obtained from the present study, it is likely that this locus has a minor role in the incidence of $\mathrm{HL}$ in the studied population. However, in order to determine the precise contribution of loci involved in HL in Iran, further studies are required on the role of DFNB22 and other loci in developing HL to find an approach for diagnosis, genetic counseling, and prevention.

\section{Conflict of Interests}

The authors have no conflict of interests.

\section{Ethical considerations}

This study was approved by Institutional Ethics Committee of Shahrekord University of Medical Sciences (93.12.5).

\section{Acknowledgment}

The authors would like to thank the families of patients for participating in this research and also the staff of the Cellular and Molecular Research Center of Shahrekord University of Medical Sciences due to their unassuming contributions. We also thank the Research Deputy of Shahrekord University of Medical Sciences for supporting the study financially with grant number 1768 .

\section{References}

1. Genç GA, Konukseven O, Muluk NB, Kirkim G, Başar FS, Tuncer $U$, et al. Features of unilateral hearing loss detected by newborn hearing screening programme in different regions of Turkey. Auris Nasus Larynx. 2013;40(3):251-9. doi: 10.1016/j. anl.2012.09.003.

2. Schrijver I. Hereditary non-syndromic sensorineural hearing loss: transforming silence to sound. J Mol Diagn. 2004;6(4):27584. doi: 10.1016/s1525-1578(10)60522-3.

3. Van Laer L, Cryns K, Smith RJ, Van Camp G. Nonsyndromic hearing loss. Ear Hear. 2003;24(4):275-88. doi: 10.1097/01. aud.0000079805.04016.03.

4. Dror AA, Avraham KB. Hearing loss: mechanisms revealed by genetics and cell biology. Annu Rev Genet. 2009;43:411-37. doi: 10.1146/annurev-genet-102108-134135.

5. Hereditary hearing loss homepage [Online]. https:// hereditaryhearingloss.org/. Accessed May 13, 2015.

6. Saadat M. Consanguineous marriages in Iranian folktales. Community Genet. 2007;10(1):38-40. doi: 10.1159/000096280.
7. Naghavi A, Nishimura C, Kahrizi K, Riazalhosseini Y, Suraki Aliabadi $\mathrm{H}$, Mahdieh $\mathrm{N}$, et al. Prevalence of GJB2 mutations among patients with autosomal recessive non syndromic hearing loss in Sistan and Baloochestan province. Zahedan Journal of Research in Medical Sciences. 2005;7(2):85-92. [Persian].

8. Lander ES, Botstein D. Homozygosity mapping: a way to map human recessive traits with the DNA of inbred children. Science. 1987;236(4808):1567-70. doi: 10.1126/science.2884728.

9. Jovine L, Park J, Wassarman PM. Sequence similarity between stereocilin and otoancorin points to a unified mechanism for mechanotransduction in the mammalian inner ear. BMC Cell Biol. 2002;3:28. doi: 10.1186/1471-2121-3-28.

10. ClinVar homepage. Available from: http://www.ncbi.nlm.nih. gov/clinvar/.

11. Grimberg J, Nawoschik S, Belluscio L, McKee R, Turck A, Eisenberg A. A simple and efficient non-organic procedure for the isolation of genomic DNA from blood. Nucleic Acids Res. 1989;17(20):8390. doi: 10.1093/nar/17.20.8390.

12. Tabatabaiefar M, Alasti F, Montazer Zohour M, Shariati L, Farrokhi E, Farhud D, et al. Genetic linkage analysis of 15 DFNB loci in a group of Iranian families with autosomal recessive hearing loss. Iran J Public Health. 2011;40(2):34-48.

13. Najmabadi H, Nishimura C, Kahrizi K, Riazalhosseini Y, Malekpour M, Daneshi A, et al. GJB2 mutations: passage through Iran. Am J Med Genet A. 2005;133A(2):132-7. doi: 10.1002/ajmg.a.30576.

14. Hashemzadeh-Chaleshtori M, Farhud D, Patton M. Familial and sporadic GJB2-related deafness in Iran: review of gene mutations. Iran J Public Health. 2007;36(1):1-14.

15. Zwaenepoel I, Mustapha M, Leibovici M, Verpy E, Goodyear R, Liu $X Z$, et al. Otoancorin, an inner ear protein restricted to the interface between the apical surface of sensory epithelia and their overlying acellular gels, is defective in autosomal recessive deafness DFNB22. Proc Natl Acad Sci U S A. 2002;99(9):62405. doi: 10.1073/pnas.082515999.

16. Shahin H, Walsh T, Rayyan AA, Lee MK, Higgins J, Dickel D, et al. Five novel loci for inherited hearing loss mapped by SNPbased homozygosity profiles in Palestinian families. Eur J Hum Genet. 2010;18(4):407-13. doi: 10.1038/ejhg.2009.190.

17. Lee K, Chiu I, Santos-Cortez RL, Basit S, Khan S, Azeem Z, et al. Novel OTOA mutations cause autosomal recessive nonsyndromic hearing impairment in Pakistani families. Clin Genet. 2013;84(3):294-6. doi: 10.1111/cge.12047.

18. Adato A, Raskin L, Petit C, Bonne-Tamir B. Deafness heterogeneity in a Druze isolate from the Middle East: novel OTOF and PDS mutations, low prevalence of GJB2 35delG mutation and indication for a new DFNB locus. Eur J Hum Genet. 2000;8(6):437-42. doi: 10.1038/sj.ejhg.5200489.

19. Ouyang XM, Yan D, Yuan HJ, Pu D, Du LL, Han DY, et al. The genetic bases for non-syndromic hearing loss among Chinese. J Hum Genet. 2009;54(3):131-40. doi: 10.1038/jhg.2009.4.

20. Najmabadi H, Kahrizi K. Genetics of non-syndromic hearing loss in the Middle East. Int J Pediatr Otorhinolaryngol. 2014;78(12):2026-36. doi: 10.1016/j.ijporl.2014.08.036.

21. De Keulenaer S, Hellemans J, Lefever S, Renard JP, De Schrijver J, Van de Voorde $\mathrm{H}$, et al. Molecular diagnostics for congenital hearing loss including 15 deafness genes using a next generation sequencing platform. BMC Med Genomics. 2012;5:17. doi: 10.1186/1755-8794-5-17.

22. Babanejad M, Fattahi Z, Bazazzadegan N, Nishimura C, Meyer N, Nikzat N, et al. A comprehensive study to determine heterogeneity of autosomal recessive nonsyndromic hearing loss in Iran. Am J Med Genet A. 2012;158A(10):2485-92. doi: 10.1002/ajmg.a.35572. 